\title{
O Teste do Degrau de Seis Minutos como Preditor de Capacidade Funcional de Acordo com o Consumo de Oxigênio de Pico em Pacientes Cardíacos
}

\author{
The Six-Minute Step Test as a Predictor of Functional Capacity according to Peak VO in Cardiac Patients
}

Luiz Eduardo Fonteles Ritt, ${ }^{1,2}$ [1] Eduardo Sahade Darzé, ${ }^{1,2}$ Gustavo Freitas Feitosa, ${ }^{1}$ Jessica Santana Porto, ${ }^{1}$ Gabriela Bastos, ${ }^{1}$ Renata Braga Linhares de Albuquerque, ${ }^{2}$ Cristiane Miura Feitosa, ${ }^{1}$ Thaissa Costa Claro, ${ }^{1}$ Eloisa Ferreira Prado, ${ }^{1}$ Queila Borges de Oliveira, ${ }^{1}$ Ricardo Stein ${ }^{3,4}{ }^{(0}$

Hospital Cárdio Pulmonar, ${ }^{1}$ Salvador, BA - Brasil

Escola Bahiana de Medicina e Saúde Pública, ${ }^{2}$ Salvador, BA - Brasil

Hospital de Clínicas de Porto Alegre, ${ }^{3}$ Porto Alegre, RS - Brasil

Universidade Federal do Rio Grande do Sul, ${ }^{4}$ Porto Alegre, RS - Brasil

\section{Resumo}

Fundamento: O teste do degrau de seis minutos (TD6) é uma forma simples de avaliar a capacidade funcional, embora tenha sido pouco estudado em pacientes com doença arterial coronariana (DAC) ou insuficiência cardíaca (IC).

Objetivo: Analisar a associação entre o TD6 e o consumo de oxigênio de pico $\left(\mathrm{VO}_{2 \text { pico }}\right)$ e desenvolver uma equação que estime o $\mathrm{VO}_{2 \text { ico }}$ com base no TD6, bem como determinar um ponto de corte para o TD6 que preveja um $\mathrm{VO}_{2 \text { ico }} \geq 20 \mathrm{~mL}$. $\mathrm{kg}^{-1} \cdot \mathrm{min}^{-1}$

Métodos: Nos 171 pacientes submetidos ao TD6 e a um teste de exercício cardiopulmonar, análises da curva ROC, de regressão e de correlação foram usadas, e um $\mathrm{p}<0,05$ foi admitido como significativo.

Resultados: A idade média foi $60 \pm 14$ anos, e $74 \%$ eram do sexo masculino. A média da fração de ejeção ventricular esquerda foi $57 \pm 16 \% ; 74 \%$ apresentavam DAC, e $28 \%$, IC. A média do VO ${ }_{2 \text { ico }}$ foi $19 \pm 6 \mathrm{~mL}^{\mathrm{k}} \mathrm{kg}^{-1}$. $\mathrm{min}^{-1}$, e o desempenho

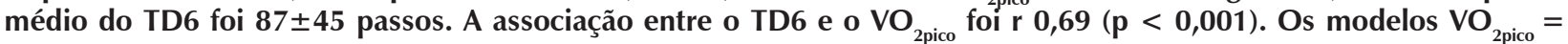
$19,6+(0,075 \times$ TD6 $)-(0,10 \times$ idade $)$ para homens e VO $\mathrm{O}_{2 \text { ico }}=19,6+(0,075 \times$ TD6 $)-(0,10 \times$ idade $)-2$ para mulheres poderiam prever o $\mathrm{VO}_{2 \text { pico }}$ com base nos resultados do TD6 ( $\mathrm{R}$ ajustado 0,$72 ; \mathrm{R}^{2}$ ajustado 0,53 ). $\mathrm{O}$ ponto de corte mais acurado para que o TD6 preveja um $\mathrm{VO}_{2 \text { pico }} \geq 20 \mathrm{~mL} . \mathrm{kg}^{-1} \cdot \mathrm{min}^{-1}$ foi de $>105$ passos [área sob a curva 0,85 ; intervalo de confiança de $95 \% 0,79-0,90 ; p<0,001]$.

Conclusão: Uma equação que preveja o $\mathrm{VO}_{2 \text { pico }}$ com base nos resultados do TD6 foi derivada, e foi encontrada uma

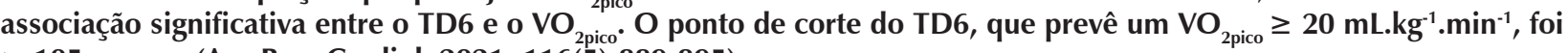
$>105$ passos. (Arq Bras Cardiol. 2021; 116(5):889-895)

Palavras-chave: Insuficiência Cardíaca; Consumo de Oxigênio; Capacidade Respiratória; Volume de Ventilação Pulmonar; Teste de Esforço.

\footnotetext{
Abstract

Background: Six-minute step test (6MST) is a simple way to evaluate functional capacity, although it has not been well studied in patients with coronary artery disease (CAD) or heart failure (HF).
}

Objective: Analyze the association between the 6MST and peak oxygen uptake $\left(V_{2}{ }_{2 p a x}\right)$ and develop an equation for estimating VO $\mathrm{F}_{2 \text { eak }}$ based on the 6MST, as well as to determine a cutoff point for the 6MST that predicts a $V O_{2 \text { peak }}^{2 p e a k} \geq 20 \mathrm{~mL} . \mathrm{Kg}^{-1} \cdot \mathrm{min}^{-1}$.

Methods: In 171 patients who underwent the 6MST and a cardiopulmonary exercise test, correlation, regression, and ROC analysis were used and a $p<0.05$ was admitted as significant.

Correspondência: Luiz Eduardo Fonteles Ritt •

Centro de Estudos Clínicos - Hospital Cárdio Pulmonar - Avenida Anita Garibaldi, 2199. CEP 40170-130, Ondina, Salvador, BA - Brasil

E-mail: luizritt@hotmail.com, lefr@cardiol.br

Artigo recebido em 11/09/2019, revisado em 16/03/2020, aceito em 08/04/2020

DOI: https://doi.org/10.36660/abc.20190624 


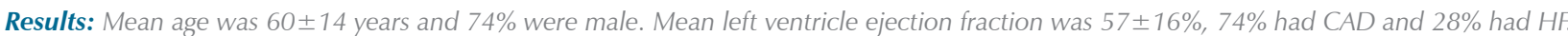
Mean $\mathrm{VO}_{2 \text { peak }}$ was $19 \pm 6 \mathrm{~mL} \cdot \mathrm{Kg}^{-1} \cdot \mathrm{min}^{-1}$ and mean $6 \mathrm{MST}$ performance was $87 \pm 45$ steps. Association between $6 \mathrm{MST}$ and VO 2 peak was $^{\mathrm{r}} 0.69$ (p $<0.001)$. The model $V O_{2 \text { peak }}=19.6+(0.075 \times 6 \mathrm{MST})-(0.10 \times$ age $)$ for men and $\mathrm{VO}_{2 \text { peak }}=19.6+(0.075 \times 6 \mathrm{MST})-(0.10 \times$ age $)-2$ for women could predict $\mathrm{VO}_{2 \text { eak }}$ based on 6MST results (adjusted $R 0.72$; adjusted $R^{2}$ 0.53). The most accurate cutoff point for $6 \mathrm{MST}$ to predict a VO

$\geq 20 \mathrm{~mL} . \mathrm{Kg}^{-1} \cdot \mathrm{min}^{-1}$ was $>105$ steps (AUC 0.85; 95\% Cl $0.79-0.90 ; p<0.001$ ).

Conclusion: An equation for predicting $\mathrm{VO}_{2 \text { eak }}$ based on 6MST results was derived, and a significant association was found between 6MST and $\mathrm{VO}_{2 \text { peak. }}$. The cutoff point for 6MST, which predicts a $V_{2 \text { eak }} \geq 20 \mathrm{~mL} \cdot \mathrm{Kg}^{-1} \cdot \mathrm{min}^{-1}$, was > 105 steps. (Arq Bras Cardiol. 2021; 116(5):889-895)

Keywords: Heart Failure; Oxygen Consumption; Respiratoty Capacity; Tidal Volume; Exercise Test.

Full texts in English - http://www.arquivosonline.com.br

\section{Introdução}

Na doença cardiovascular, a capacidade funcional está diretamente relacionada ao prognóstico. ${ }^{1} \mathrm{O}$ desempenho funcional, conforme determinado pelo consumo de oxigênio de pico $\left(\mathrm{VO}_{2 \text { pico }}\right)$ e medido por um teste de exercício cardiopulmonar (TECP), é o padrão-ouro e é utilizado para determinar o prognóstico de insuficiência cardíaca (IC) e seleção de transplante cardíaco, bem como para avaliar a resposta terapêutica. ${ }^{2-4}$ Pacientes com $\mathrm{VO}_{2 \text { pico }}$ abaixo de $15 \mathrm{~mL} \cdot \mathrm{kg}^{-1} \cdot \mathrm{min}^{-1}$ apresentam um perfil prognóstico pior, e aqueles com $\mathrm{VO}_{2 \text { pico }}$ acima de $20 \mathrm{~mL} \cdot \mathrm{kg}^{-1} \cdot \mathrm{min}^{-1}$ apresentam um perfil prognóstico melhor, independentemente da etiologia da IC e da função ventricular., ${ }^{5,6}$ Embora amplamente utilizado e validado, o TECP não está disponível na maioria dos centros, pois o equipamento é caro e é necessário que um médico especializado administre o teste e interprete seus resultados.

Uma alternativa ao TECP é o teste de caminhada de seis minutos (TC6), o qual é bem validado e apresenta boa correlação com o TECP em pacientes com cardiomiopatia. ${ }^{7} \mathrm{No}$ entanto, o TC6 requer um longo corredor (com pelo menos 30 metros), o que pode limitar seu uso na prática comum.

O teste do degrau de seis minutos (TD6) é um teste simples no qual o paciente sobe e desce uma escada de 2 degraus por 6 minutos em cadência livre, e o número de passos é contabilizado. Não requer equipamentos sofisticados nem espaços grandes. Embora estudado em pacientes com doença pulmonar crônica e em indivíduos normai,8-11 não há dados sobre o desempenho do TC6 em pacientes cardíacos.

Os objetivos deste estudo foram: (1) analisar a associação entre o TD6 e o $\mathrm{VO}_{2 \text { pico' }}$ (2) desenvolver uma equação para estimar o $\mathrm{VO}_{2 \text { piсо }}$ com base nos resultados do TD6 e (3) determinar um ponto de corte para a categoria de baixo risco no TD6 $\left(\mathrm{VO}_{2 \text { piсо }} \geq 20 \mathrm{~mL} \cdot \mathrm{kg}^{-1} \cdot \mathrm{min}^{-1}\right)$.

\section{Métodos}

Neste estudo transversal, avaliamos pacientes encaminhados para reabilitação cardíaca entre maio de 2014 e setembro de 2017 que, conforme o protocolo clínico, foram submetidos a TECP limitado a sintomas e TD6 como avaliação basal no programa de reabilitação cardíaca do Hospital Cárdio Pulmonar, em Salvador, Brasil.

Os critérios de inclusão foram pacientes maiores de 18 anos com diagnóstico de doença arterial coronariana (DAC) ou IC, caracterizadas por infarto agudo do miocárdio prévio, angioplastia coronariana/implante de stent pós- cirurgia cardíaca ou vascular ou pacientes com dispositivos implantáveis, como marca-passos ou desfibriladores cardíacos. Esses indivíduos foram encaminhados ao programa de reabilitação cardíaca e submetidos a avaliação inicial com cardiologista e fisioterapeuta. O diagnóstico de DAC e/ou IC foi estabelecido pelo histórico médico (infarto agudo do miocárdio, DAC estável, revascularização do miocárdio ou angioplastia ou sintomas de dispneia ou angina), anormalidades eletrocardiográficas (ondas Q patológicas) e anormalidades ecocardiográficas (disfunção ventricular e anormalidades segmentares).

O critério de exclusão foi incapacidade de realizar o TECP ou o TD6. Pacientes com sintomas de angina ou isquemia em estágio inferior ao limiar anaeróbio também foram excluídos por não terem sido submetidos ao TD6.

Os dados clínicos e demográficos foram obtidos da avaliação cardiológica inicial no dia do TECP, incluindo o ecocardiograma mais recente (nos últimos 3 meses). O TECP e o TD6 foram aplicados separadamente, com 2 a 7 dias de intervalo.

O TD6 foi realizado em um degrau de $20 \mathrm{~cm}$ de altura coberto com borracha antiderrapante. Os pacientes foram instruídos a subir e descer o degrau o mais rápido possível por 6 minutos, sem usar os braços para se apoiar; pausas para descanso eram permitidas durante os 6 minutos.

O TECP limitado a sintomas foi realizado em uma esteira com um analisador de gases (Cortex, Leipzig, Alemanha) com medidas a cada respiração. Foi utilizado um protocolo de rampa individualizado baseado na classe funcional de cada paciente, com duração da fase de exercício direcionado entre 8 e 12 minutos. Os dados ventilatórios coletados foram tabulados e analisados em intervalos de 10 segundos.

Aspectos éticos: o protocolo do estudo foi aprovado pelo Comitê de Ética em Pesquisa Celso Figueiroa do Hospital Santa Izabel (processo 1.711.505). O estudo foi conduzido de acordo com a legislação nacional e internacional de pesquisa em humanos, incluindo a Declaração de Helsinque e a resolução 466/12 do Conselho Nacional de Saúde do Brasil. O consentimento informado foi dispensado, uma vez que o estudo utilizou apenas dados de prontuários médicos.

\section{Análise Estatística}

Todas as análises foram realizadas no software SPSS, versão 25.0. As variáveis contínuas foram apresentadas como média \pm desvio padrão para a distribuição paramétrica. O teste de Shapiro-Wilk e a inspeção visual dos histogramas 
foram utilizados para determinar a normalidade. As variáveis categóricas foram apresentadas em número ou porcentagem. A correlação de Pearson foi aplicada para determinar associações entre variáveis contínuas, e os gráficos de BlandAltman foram utilizados para analisar sua concordância. Análises de regressão linear univariada e multivariada (após análise das suposições adequadas) foram realizadas para determinar a previsão de $\mathrm{VO}_{2 \text { pico }}$ do modelo com base no TD6, o qual foi controlado para idade, fração de ejeção, sexo, presença de DAC ou IC e peso. A análise da curva ROC foi aplicada para determinar os melhores pontos de corte para prever $\mathrm{VO}_{2 \text { pico }} \geq 20 \mathrm{~mL} \cdot \mathrm{kg}^{-1} \cdot \mathrm{min}^{-1}$. Um valor de $\mathrm{p}$ de $<0,05$ foi considerado estatisticamente significativo.

\section{Resultados}

A amostra total consistiu em 171 indivíduos. Suas características clínicas e demográficas são apresentadas na Tabela 1. A maioria dos pacientes apresentou classe funcional I ou II da New York Heart Association (NYHA) com $\mathrm{VO}_{2 \text { ico }}$ médio de $19 \pm 6 \mathrm{~mL} \cdot \mathrm{kg}^{-1} \cdot \mathrm{min}^{-1}$.

A associação entre o TD6 e o $\mathrm{VO}_{2 \text { pico }}$ é apresentada na Figura 1; o índice de correlação $r$ foi 0,69 (IC95\% 0,60 $0,78 ; p<0,001)$, e o de $R^{2}$ foi 0,47 . A análise do gráfico

Tabela 1 - Características clínicas e demográficas gerais da população

\begin{tabular}{|c|c|}
\hline Variável & Resultado \\
\hline Sexo masculino \% (n) & $74 \%(121)$ \\
\hline Idade (anos) & $60 \pm 14$ \\
\hline DAC \% (n) & $74 \%(121)$ \\
\hline Insuficiência cardíaca \% (n) & $28 \%(47)$ \\
\hline Valvulopatias \% (n) & $13 \%(22)$ \\
\hline Diabetes \% (n) & $25 \%(44)$ \\
\hline Hipertensão \% (n) & $62 \%(102)$ \\
\hline NYHA I, II, III\% & $53 \% / 24 \% / 10 \%$ \\
\hline Inibidor de ECA-BRA \% (n) & $65 \%(110)$ \\
\hline Betabloqueador \% (n) & $77 \%(130)$ \\
\hline Estatinas \% (n) & $75 \%(128)$ \\
\hline Fração de ejeção (\%) & $57 \pm 16$ \\
\hline $\mathrm{VO}_{2 \text { pico }}\left(\mathrm{mL} \cdot \mathrm{kg}^{-1} \cdot \mathrm{min}-1\right)$ & $19 \pm 6$ \\
\hline $\mathrm{VO}_{2}$ no limiar anaeróbio $\left(\mathrm{mL} \cdot \mathrm{kg}^{-1} \cdot \mathrm{min}^{-1}\right)$ & $12.6 \pm 3$ \\
\hline RER & $1.12 \pm 0.8$ \\
\hline Inclinação VENCOO & $36 \pm 10$ \\
\hline TD6 (passos) & $85 \pm 47$ \\
\hline \multicolumn{2}{|c|}{$\begin{array}{l}\text { BRA: bloqueador dos receptores da angiotensina; DAC: doença } \\
\text { arterial coronariana; ECA: enzima conversora da angiotensina; } \\
\text { NYHA: New York Heart Association; RER: razão de troca } \\
\text { respiratória; VENCO }{ }_{2:} \text { relação entre a ventilação e a produção de } \\
\text { dióxido de carbono. }\end{array}$} \\
\hline
\end{tabular}

de Bland-Altman é apresentada na Figura 2; a concordância estava distante do limite de referência superior ou inferior em apenas cinco pacientes.

$\mathrm{Na}$ análise multivariada, a idade, o sexo e os resultados do TD6 foram preditores independentes do $\mathrm{VO}_{2 \text { pico }}$ (Tabela 2). As equações para a estimativa do $\mathrm{VO}_{2 \text { pico }}$ com base no TD6 foram: $\mathrm{VO}_{2 \text { pico }}=19,6+(0,075 \times \mathrm{TD} 6)-(0,10 \times$ idade $)$ para homens e $\mathrm{VO}_{\text {2рісо }}=19,6+(0,075 \times$ TD6 $)-(0,10 \times$ idade $)-2$ para mulheres. O r ajustado do modelo final foi 0,72 , e o $\mathrm{R}^{2}$ ajustado foi 0,53 .

A curva ROC para o TD6 como preditor de $\mathrm{VO}_{2 \text { pico }} \geq 20$ $\mathrm{mL} \cdot \mathrm{kg}^{-1} \cdot \mathrm{min}^{-1}$ é apresentada na Figura 3. O ponto de corte mais acurado para que o TD6 preveja $\mathrm{VO}_{2 \text { pico }} \geq 20 \mathrm{~mL} . \mathrm{kg}^{-1}$. $\min ^{-1}$ foi $>105$ passos (área sob a curva 0,85; IC95\% 0,79$0,90 ; p<0,001)$.

\section{Discussão}

A capacidade funcional é um dos parâmetros clínicos mais importantes para a avaliar a capacidade funcional. ${ }^{1}$ O comprometimento funcional está relacionado a pior prognóstico, independentemente do diagnóstico ou cenário clínico. ${ }^{1,7}$ A aptidão cardiorrespiratória (ACR) pode ser estimada por diversos métodos, embora o TECP seja o único método que permite determinação direta com base no $\mathrm{VO}_{2 \text { pico }}$. Uma vez que o TECP requer equipamento específico e equipe médica bem treinada, uma medição indireta e acurada da capacidade funcional é muito desejável. É importante que sejam validados formulários alternativos mais simples para a avaliação de ACR, já que podem ser aplicados de forma mais ampla.

Em uma população de pacientes com DAC e IC, demonstramos que o TD6 apresentou boa correlação com o $\mathrm{VO}_{2 \text { ico, }}$ conforme medido pelo TCEP. Também conseguimos derivar uma equação para prever o $\mathrm{VO}_{2 \text { pico }}$ com base nos resultados do TD6, bem como para determinar um ponto de corte para o número de passos necessários para identificar pacientes de baixo risco (valor mínimo do $\mathrm{VO}_{2 \text { pico }}$ de $\left.20 \mathrm{~mL} \cdot \mathrm{kg}^{-1} \cdot \mathrm{min}^{-1}\right)$.

\section{Teste do Degrau na Cardiologia}

Os testes do degrau não são uma ferramenta nova na cardiologia. Na década de 1930, Master et al., ${ }^{8}$ utilizaram um teste de escada de um degrau em um protocolo de 2 minutos para observar eletrocardiogramas de exercício. Esse foi o precursor dos atuais testes de esforço com ergômetros. O teste do degrau de Master era amplamente utilizado como teste provocativo para isquemia coronariana, mas não era rotineiramente utilizado como preditor de ACR/capacidade funcional e prognóstico. O principal objetivo do TD6 como teste submáximo é determinar a ACR e não diagnosticar isquemia coronariana. Assim como o TC6, o TD6 é seguro e pode ser realizado em esforço submáximo, embora com um gasto energético um pouco maior.

\section{Capacidade Funcional como Sinal Vital}

A capacidade funcional pode ser considerada um sinal vital e deve ser avaliada em todas as consultas médicas. ${ }^{9,10}$ Pode ser 


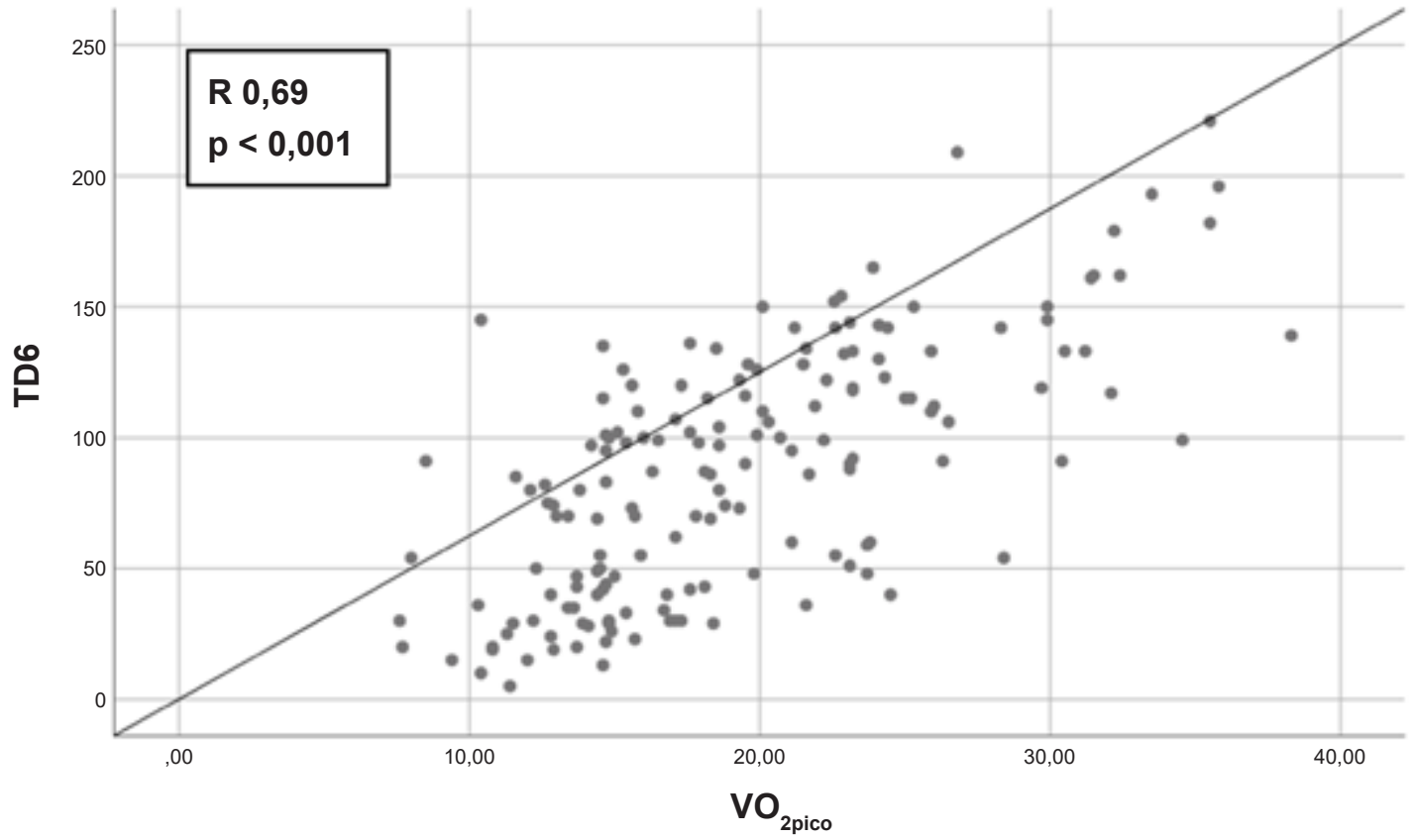

Figura 1 - Associação entre o TD6 e o $\mathrm{VO}_{2 \text { pico" }}$

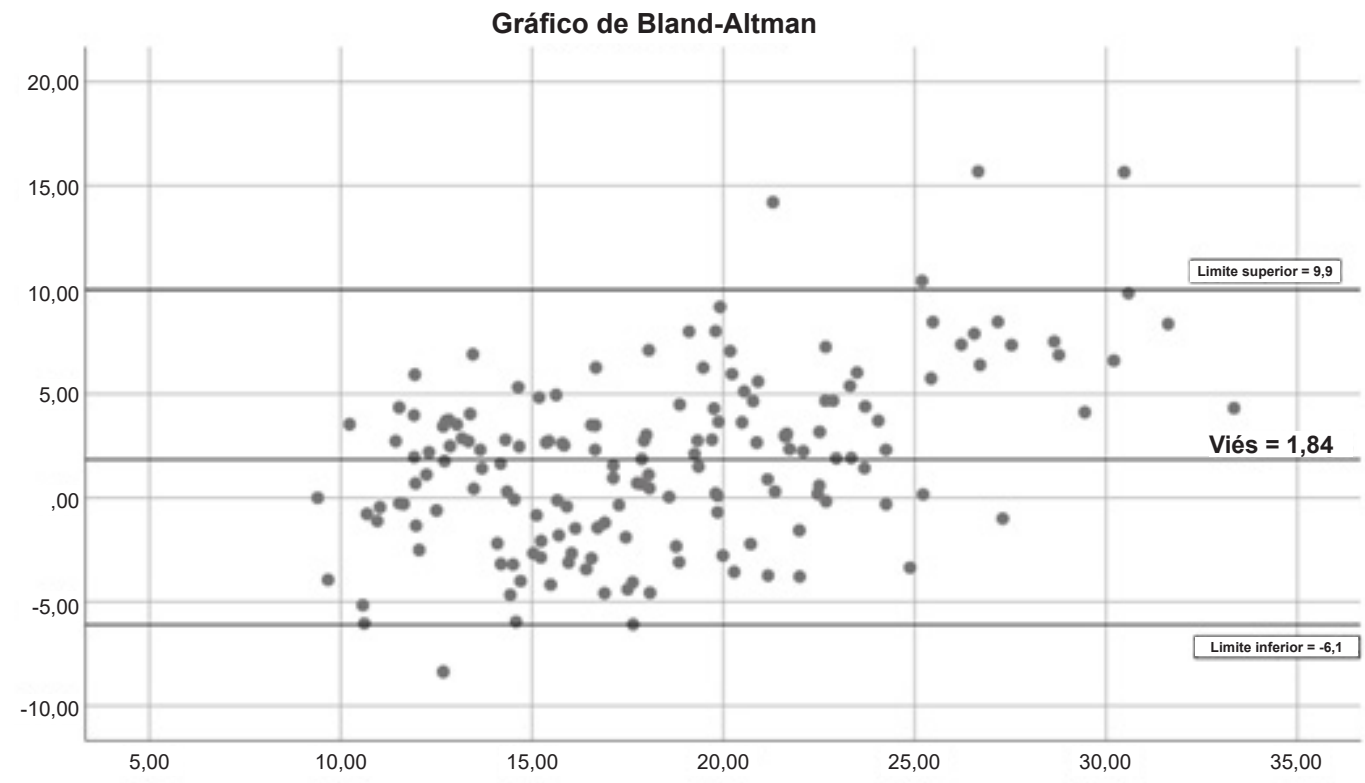

Figura 2 - Gráfico de Bland-Altman para $\mathrm{VO}_{2 \text { pico }}$ previsto vs. Determinado. 


\section{Artigo Original}

\begin{tabular}{lccc}
\hline \multicolumn{4}{l}{ Tabela 2 - Modelo final de regressão linear múltipla para predição do VO $_{2 \text { pico }}$ com base no TD6 } \\
\hline Variável & Beta & Beta IC95\% & p \\
\hline TD6 & 0,075 & $(0,06)-(0,09)$ & $<0,001$ \\
\hline Idade (anos) & $-0,10$ & $(-0,16)-(-0,5)$ & $<0,001$ \\
\hline Sexo feminino & $-2,0$ & $(-3,6)-(-0,33)$ & 0,02 \\
\hline Constante & 19,6 & $(15,2)-(24,1)$ & $<0,001$ \\
\hline
\end{tabular}

Ajustado para idade, fração de ejeção, doença arterial coronariana, insuficiência cardíaca e peso. IC: intervalo de confiança; TD6: teste do degrau de seis minutos.

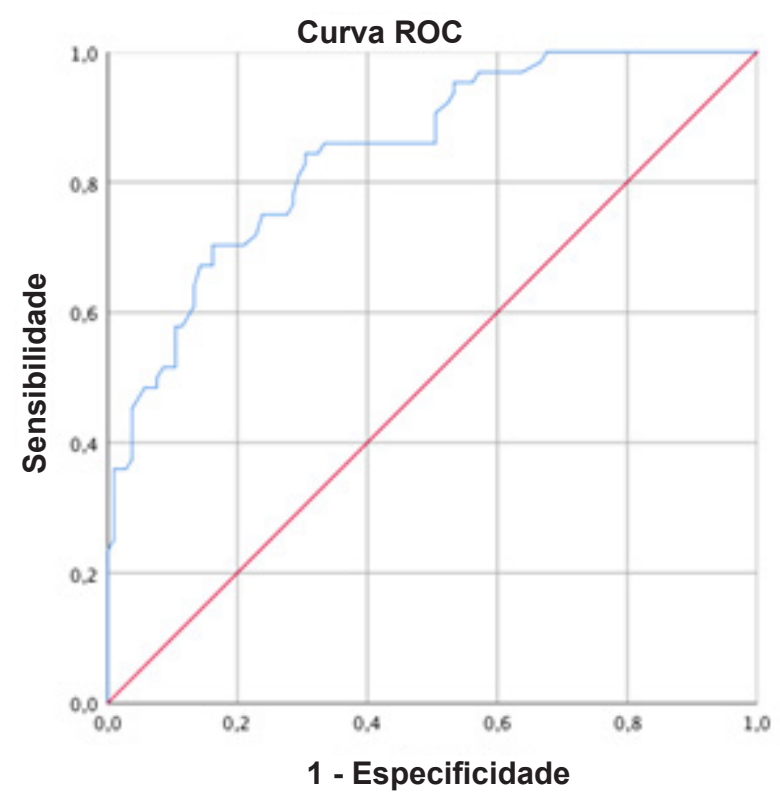

Figura 3 - Curva ROC para que o TD6 preveja um VO ${ }_{20 i c 0} \geq 20 \mathrm{~mL} \cdot \mathrm{kg}^{1}$. $\mathrm{min}^{-1}$. Área sob a curva 0.85 (IC95\% 0,79 - 0,90) p < 0,001.

prevista por testes de exercício regulares ou testes funcionais submáximos, como o TC6; no entanto, o TCEP é a única forma de avaliar e determinar diretamente a capacidade funcional. Com base em estudos clássicos em pacientes cardíacos, um $\mathrm{VO}_{\text {2pico }}$ acima de $20 \mathrm{~mL} \cdot \mathrm{kg}^{-1} \cdot \mathrm{min}^{-1}$ é um marcador de bom prognóstico, independentemente de outros parâmetros. Por outro lado, aqueles com $\mathrm{VO}_{2 \text { pico }}$ abaixo de $12 \mathrm{~mL} \cdot \mathrm{kg}^{-1} \cdot \mathrm{min}^{-1} \mathrm{e}$ IC podem ser considerados candidatos a transplante cardíaco. ${ }^{5,6}$

Como alternativa ao TCEP, o TC6 foi validado e é utilizado para avaliação prognóstica em diferentes doenças. ${ }^{11}$ É de fácil reprodução e pode ser relacionado ao desfecho, mas a necessidade de um espaço grande impede seu uso no consultório, por exemplo. Portanto, um teste que consiga estimar a capacidade funcional mesmo em espaço pequeno sem a necessidade de equipamentos sofisticados é de grande utilidade. É importante destacar que o TD6 foi previamente comparado ao TC6 em uma população sem doenças pulmonares ou cardíacas, apresentando boa correlação. ${ }^{12}$

O teste do degrau de seis minutos é uma forma simples de predizer capacidade funcional
O TD6 é um teste simples que não necessita de muito espaço. Pode ser realizado em um consultório médico ou por outros profissionais da saúde. O teste foi usado previamente em pacientes com doença pulmonar crônica, mas ainda não foi validado em pacientes cardíacos.

Em pacientes com doença pulmonar obstrutiva crônica, um ponto de corte de $<78$ passos foi associado a pior prognóstico. ${ }^{13} \mathrm{Em}$ uma população saudável com média de idade de 39 anos, a contagem média de passos foi $149 \pm 34{ }^{14}$

De acordo com nossos dados, o TD6 apresenta acurácia aceitável para prever o $\mathrm{VO}_{2 \text { pico }}$ em uma amostra de pacientes com DAC/IC, e os profissionais de saúde podem querer usar esses resultados em sua prática clínica.

Observamos que o ponto de corte de > 105 passos está relacionado ao alcance de um $\mathrm{VO}_{2 \text { pico }}$ acima de $20 \mathrm{~mL} . \mathrm{kg}^{-1}$. $\mathrm{min}^{-1}$. Esse ponto de corte pode ser útil, por exemplo, quando o TCEP não estiver disponível. Além disso, se o paciente consegue subir mais de 105 degraus, o TCEP pode não ser necessário, pois estima-se um $\mathrm{VO}_{2 \text { pico }}$ acima de $20 \mathrm{~mL} \cdot \mathrm{kg}^{-1} \cdot \mathrm{min}^{-1}$. 
As estimativas de capacidade funcional baseadas em atividades de rotina são imprecisas e não foram validadas diretamente por meio de dados do TCEP ${ }^{15}$ embora essa estratégia ainda seja utilizada quando estimativas imediatas são necessárias, mesmo para avaliação em série. Assim, o TD6 pode ser aplicado de forma fácil e rápida, porém com mais segurança no que se refere à determinação da capacidade funcional.

\section{Limitações}

O presente estudo apresenta algumas limitações. Uma amostra maior e uma validação prospectiva dos resultados em outras populações devem ser consideradas. Nossa população consistiu em pacientes com DAC e/ou IC, os quais foram analisados em conjunto. Pode ser interessante analisar esses fenótipos separadamente. Para atenuar a influência do diagnóstico clínico na realização do teste, controlamos a análise multivariada para o diagnóstico de IC ou DAC e constatamos que o diagnóstico não influenciou o resultado. Também controlamos a análise para a fração de ejeção. Como a DAC é a causa mais prevalente de IC e a capacidade funcional é um fator prognóstico independente para ambos, a existência de um teste e um único ponto de corte que possa ser aplicado em um espectro mais amplo de doenças cardíacas pode ser de grande utilidade, já que o TD6 pode ser mais bem aplicado em triagem e acompanhamento.

A análise dos gráficos de Bland-Altman mostrou que a concordância foi considerada distante dos limites de controle superior ou inferior em apenas cinco pacientes. Desses, quatro apresentaram $\mathrm{VO}_{2 \text { ico }}$ acima de $20 \mathrm{~mL} \cdot \mathrm{kg}^{-1} \cdot \mathrm{min}^{-1}$, e o valor previsto no TD6 também foi maior de $20 \mathrm{~mL} \cdot \mathrm{kg}^{-1} \cdot \mathrm{min}^{-1}$. Dessa forma, os quatro pacientes não seriam erroneamente classificados como de menor risco do que o esperado. Em um paciente, o $\mathrm{VO}_{2 \text { pico }}$ previsto pelo TD6 foi maior do que o medido. Ao analisar esse caso, observamos que a razão de troca respiratória do TCEP apresentou compatibilidade de apenas 0,94 com um esforço submáximo, causada pela má adaptação à esteira e à máscara. O mesmo paciente subiu e desceu 91 degraus em 6 minutos. Pode-se entender que o TD6 é mais adequado como ferramenta de triagem e não como um substituto do TCEP. Portanto, embora sejam correlacionados, o TCEP ainda é necessário em casos em que a capacidade funcional precisa ser determinada com exatidão.

No momento, não temos dados de desempenho do TC6 para esses indivíduos, embora uma correlação entre os testes nesses pacientes possa ser útil. Estudos correlacionando o desempenho do TD6 em termos de desfechos clínicos devem fornecer mais informações sobre os melhores pontos de corte. Por fim, embora o TCEP $\mathrm{VO}_{2 \text { pico }}$ seja o padrãoouro para a avaliação funcional, é possível que o TD6 possa fornecer algumas implicações prognósticas, de acordo com os resultados.

\section{Conclusão}

Foi derivada uma equação capaz de prever o $\mathrm{VO}_{2 \text { pico }} \mathrm{com}$ base nos resultados do TD6, e foi encontrada associação significativa entre o TD6 e o $\mathrm{VO}_{2 \text { pico }}$. O ponto de corte do TD6, que prevê um $\mathrm{VO}_{2 \text { pico }} \geq 20 \mathrm{~mL} \cdot \mathrm{kg}^{-1} \cdot \mathrm{min}^{-1}$, foi $>105$ passos.

\section{Agradecimentos}

Foi fornecido apoio financeiro parcial pelo FIPE-HCPA (Fundo de Amparo à Pesquisa do Hospital de Clínicas de Porto Alegre).

\section{Contribuição dos Autores}

Concepção e desenho da pesquisa: Ritt LE, Porto JS, Bastos G, Feitosa CM, Claro TC, Prado EF, Oliveira QB, Stein R; Obtenção de dados: Ritt LE, Feitosa GF, Porto JS, Bastos G, Albuquerque RBL, Feitosa CM, Claro TC, Prado EF, Oliveira QB, Stein R; Análise e interpretação dos dados: Ritt LE, Darzé ES, Feitosa GF, Porto JS, Bastos G, Prado EF, Stein R; Análise estatística: Ritt LE, Darzé ES, Porto JS, Bastos G, Albuquerque RBL, Oliveira QB, Stein R; Obtenção de financiamento: Ritt LE, Stein R; Redação do manuscrito: Ritt LE, Darzé ES, Feitosa GF, Porto JS, Bastos G, Albuquerque RBL, Stein R; Revisão crítica do manuscrito quanto ao conteúdo intelectual importante: Ritt LE, Darzé ES, Feitosa GF, Porto JS, Bastos G, Feitosa CM, Claro TC, Prado EF, Oliveira QB, Stein R.

\section{Potencial Conflito de Interesses}

Declaro não haver conflito de interesses pertinentes.

\section{Fontes de Financiamento}

O presente estudo não teve fontes de financiamento externas.

\section{Vinculação Acadêmica}

Não há vinculação deste estudo a programas de pósgraduação.

\section{Referências}

1. Forman DE, Arena R, Boxer R, Dolansky MA, Eng JJ, FlegJL, et al. Prioritizing Functional Capacity as a Principal End Point for Therapies Oriented to Older Adults with Cardiovascular Disease: A Scientific Statement for Healthcare Professionals from the American Heart Association. Circulation. 2017;135(16):e894-918.

2. Task Force of the Italian Working Group on Cardiac Rehabilitation and Prevention. Statement on cardiopulmonary exercise testing in chronic heart failure due to left ventricular dysfunction: recommendations for performance and interpretation Part III: Interpretation of cardiopulmonary exercise testing in chronic heart failure and future. Eur J Cardiovasc Prev Rehabil. 2006;13(4):485-94.
3. Fletcher GF, Ades PA, Kligfield P, Arena R, Balady GJ, Bittner VA, et al. Exercise standards for testing and training: A scientific statement from the American heart association. Circulation. 2013;128(8):873-934.

4. Malhotra R, Bakken K, D'Elia E, Lewis GD. Cardiopulmonary Exercise Testing in Heart Failure. JACC Hear Fail. 2016;4(8):607-16.

5. Myers J, Gullestad L, Vagelos R, Bellin D, Ross H, Fowler MB. Cardiopulmonary exercise testing and prognosis in severe heart failure: 14 mL/kg/min revisited. Am Heart J. 2000;139(1 I):78-84. 


\section{Artigo Original}

6. Mancini DM, Eisen H, Kussmaul W, Mull R, Edmonds LH, Wilson JR Value of pico exercise oxygen consumption for optimal timing of cardiac transplantation in ambulatory patients with heart failure. Circulation. 1991;83(3):778-86.

7. Arena R, Myers J, Williams MA, Gulati M, Kligfield P, Balady GJ, et al. Assessment of functional capacity in clinical and research settings: A scientific statement from the American Heart Association committee on exercise, rehabilitation, and prevention of the council on clinical cardiology and the council on cardiovascular n. Circulation. 2007;116(3):329-43.

8. Master A, Oppenheimer E. A simple exercise tolerance test for circulatory efficiency with standard tables for normal individuals. Vol. 177, Am J Med Sci.. 1929. p. 223-42.

9. Peterson PN, Magid DJ, Ross C, Ho PM, Rumsfeld IS, Lauer MS, Lyons EE, Smith SS MF. Association of Exercise Capacity on Treadmill With Future Cardiac Events in Patients Referred for Exercise Testing. 2014;168(2):174-9.

10. Kodama S, Saito K, Tanaka S, Maki M, Yachi Y, Asumi M, Sugawara A, Totsuka K, Shimano H, Ohashi Y, Yamada N SH. Cardiorespiratory Fitness as a Quantitative Predictor of All-Cause Mortality and Cardiovascular Events. J Am Med Assoc. 2009;301(19):2024-35.
11. ATS. Guidelines for the six-minute walk test. Am J Respir Crit Care Med. 2002;166(1):111-7.

12. da Costa CH, da Silva KM, Maiworm A, Raphael Y, Parnayba J, Da Cal M, et al. Can we use the 6-minute step test instead of the 6-minute walking test? An observational study. Physiother (United Kingdom) [Internet]. 2017;103(1):48-52. Available from: http://dx.doi.org/10.1016/j. physio.2015.11.003

13. Pessoa B V., Arcuri JF, Labadessa IG, Costa JNF, Sentanin AC, Di Lorenzo VAP. Validity of the six-minute step test of free cadence in patients with chronic obstructive pulmonary disease. Brazilian J Phys Ther [Internet]. 2014;18(3):228-36. Available from: http://www.scielo.br/scielo. php?script=sci_arttext\&pid=S1413-35552014000300228\&lng=en\&nr $\mathrm{m}=$ iso\&tlng $=\mathrm{em}$

14. Arcuri JF, Borghi-Silva A, Labadessa IG, Sentanin AC, Candolo C PDLV. Validity and reliability of the 6-minute step test in healthy individuals: $\mathrm{A}$ Cross-sectional study. Clin J Sport Med. 2016;26(1):69-75.

15. Ritt LEF, Stein R, Ribeiro DS, Ribeiro RS, Pilar I, Borges Q, et al. Low Concordance Between the Nyha Class and Cardiopulmonary Exercise Test Variables in Heart Failure Patients. J Am Coll Cardiol [Internet]. 2019;73(9):1828. Available from: http://dx.doi.org/10.1016/S07351097(19)32434-9 